\title{
Periodic Wave Solutions and Their Limit Forms of the Modified Novikov Equation
}

\author{
Qing Meng ${ }^{1}$ and Bin $\mathrm{He}^{2}$ \\ ${ }^{1}$ Department of Physics, Honghe University, Mengzi, Yunnan 661100, China \\ ${ }^{2}$ College of Mathematics, Honghe University, Mengzi, Yunnan 661100, China \\ Correspondence should be addressed to Bin He; 2468mq@163.com
}

Received 16 December 2014; Accepted 1 April 2015

Academic Editor: K. M. Liew

Copyright (c) 2015 Q. Meng and B. He. This is an open access article distributed under the Creative Commons Attribution License, which permits unrestricted use, distribution, and reproduction in any medium, provided the original work is properly cited.

\begin{abstract}
The modified Novikov equation $u_{t}-u_{t x x}+(b+1) u^{2} u_{x}=b u u_{x} u_{x x}+u^{2} u_{x x x}$ is studied by using the bifurcation theory of dynamical system and the method of phase portraits analysis. The existences, dynamic properties, and limit forms of periodic wave solutions for $b$ being a negative even are investigated. All possible exact parametric representations of the different kinds of nonlinear waves also are presented.
\end{abstract}

\section{Introduction}

The Novikov equation

$$
u_{t}-u_{x x t}+4 u^{2} u_{x}=3 u u_{x} u_{x x}+u^{2} u_{x x x}
$$

was discovered by Novikov in a symmetry classification of nonlocal PDEs with quadratic or cubic nonlinearity [1]. The perturbative symmetry approach yields necessary conditions for a PDE to admit infinitely many symmetries. Using this approach, Novikov was able to isolate (1) and find its first few symmetries, and he subsequently found a scalar Lax pair for it (also see [2]) and then proved that the equation is integrable. Hone and Wang [3] have shown that (1) admits peakon solutions like the $\mathrm{CH}$ and the DP equations. Jiang and $\mathrm{Ni}$ [4] have shown that (1) possesses the blowup phenomenon. The existence and uniqueness of global weak solutions for (1) were studied in [5]. Bozhkov et al. [6] found the Lie point symmetries of (1) and demonstrate that it is strictly self-adjoint. Li [7] obtained exact cuspon wave solution and compactons and found that the corresponding traveling system of (1) has no one-peakon solution. The Cauchy problem of (1) was investigated in $[8,9]$.

The modified Novikov equation reads as

$$
u_{t}-u_{t x x}+(b+1) u^{2} u_{x}=b u u_{x} u_{x x}+u^{2} u_{x x x}
$$

where $b$ is a real parameter. Clearly, letting $b=3,(2)$ becomes the Novikov equation (1). Lai and $\mathrm{Wu}$ [10] considered the local strong and weak solutions of (2). The global solution and blow-up phenomena of (2) were investigated in [11]. The Cauchy problem of (2) was studied in $[12,13]$.

In this paper, we consider the existences, dynamic properties, and limit forms of periodic wave solutions of (2) for $b$ being a negative even using the bifurcation theory of dynamical system and the method of phase portraits analysis $[7,14]$, we also will present some new explicit exact solutions of (2).

Using transformation

$$
u(x, t)=\phi(\xi)=\phi(x-c t)
$$

where $c(\neq 0)$ is the wave speed, (2) can be rewritten as

$$
-c \phi^{\prime}+c \phi^{\prime \prime \prime}+(b+1) \phi^{2} \phi^{\prime}=b \phi \phi^{\prime} \phi^{\prime \prime}+\phi^{2} \phi^{\prime \prime \prime},
$$

where "'l" is the derivative with respect to $\xi$.

Integrating (4) once, it follows that

$$
\begin{aligned}
\left(\phi^{2}-c\right) \phi^{\prime \prime}= & -c \phi+\frac{1}{3}(b+1) \phi^{3}-\frac{1}{2}(b-2) \phi\left(\phi^{\prime}\right)^{2} \\
& +\frac{1}{2}(b-2) \int\left(\phi^{\prime}\right)^{2} d \phi .
\end{aligned}
$$


Let $y=\phi^{\prime}$, and then (5) becomes

$$
\begin{aligned}
\left(\phi^{2}-c\right) \frac{d y^{2}}{d \phi}= & -2 c \phi+\frac{2}{3}(b+1) \phi^{3}-(b-2) \phi y^{2} \\
& +(b-2) \int y^{2} d \phi .
\end{aligned}
$$

Differentiating both sides of (6) with respect to $\phi$, we have

$$
\left(\phi^{2}-c\right) \frac{d^{2} y^{2}}{d \phi^{2}}+b \phi \frac{d y^{2}}{d \phi}=-2 c+2(b+1) \phi^{2} .
$$

It implies that

$$
\frac{d y^{2}}{d \phi}=2 \phi+A\left(\phi^{2}-c\right)^{-b / 2}
$$

where $A$ is the integral constant.

For simplicity, we only consider the special case $b=-2 m$ and $m \in Z^{+}$in this paper. For this special case, (2) becomes

$$
u_{t}-u_{t x x}+(1-2 m) u^{2} u_{x}=-2 m u u_{x} u_{x x}+u^{2} u_{x x x}
$$

and (8) can be rewritten as

$$
\frac{d y^{2}}{d \phi}=2 \phi+A\left(\phi^{2}-c\right)^{m}
$$

We see from (10) that

$$
y^{2}=\phi^{2}+A \int\left(\phi^{2}-c\right)^{m} d \phi+2 h,
$$

where $h$ is an integral constant. Thus, the function

$$
H(\phi, y)=\frac{1}{2} y^{2}-\frac{1}{2} \phi^{2}-\frac{1}{2} A \int\left(\phi^{2}-c\right)^{m} d \phi=h
$$

is a first integral of (9). The dynamics of (9) is equivalent to the system

$$
\begin{aligned}
& \frac{d \phi}{d \xi}=\frac{\partial H}{\partial y}=y, \\
& \frac{d y}{d \xi}=-\frac{\partial H}{\partial \phi}=\phi+\frac{1}{2} A\left(\phi^{2}-c\right)^{m},
\end{aligned}
$$

where $A \neq 0$; otherwise system (13) becomes a linear system.

For a fixed $h$, the level curve $H(\phi, y)=h$ defined by (12) determines a set of invariant curves of system (13) which contains different branches of curves. As $h$ is varied, it defines different families of orbits of (13) with different dynamical behaviors.

The remainder of this paper is organized as follows: In Section 2, we consider bifurcation sets and phase portraits of (13). Existences and limit forms of periodic wave solutions of (9) are stated in Section 3. Some explicit exact traveling wave solutions of (9) are presented in Section 4. A short conclusion will be given in Section 5 .

\section{Bifurcation Analysis of (13)}

Obviously, the equilibrium point of system (13) is just the intersection point of the straight line $y=\phi$ and the curve defined by $y=-(1 / 2) A\left(\phi^{2}-c\right)^{m}$ (see Figures $\left.1(\mathrm{a})-1(\mathrm{~h})\right)$. Clearly, system (13) does not have any equilibrium point when $c<(1-2 m)\left(A m(2 m)^{m-1}\right)^{2 /(1-2 m)}$. There exists only one equilibrium point $\left(\phi_{0}, 0\right)$ of (13) satisfying $\phi_{0}+(1 / 2) A\left(\phi_{0}^{2}-\right.$ $c)^{m}=0$ when $c=(1-2 m)\left(A m(2 m)^{m-1}\right)^{2 /(1-2 m)}$, and $\phi_{0}>0$ when $A<0, \phi_{0}<0$ when $A>0$. System (13) has two equilibrium points $\left(\phi_{1}, 0\right),\left(\phi_{2}, 0\right)$ satisfying $\phi_{i}+(1 / 2) A\left(\phi_{i}^{2}-\right.$ $c)^{m}=0, i=1,2$, when $c>(1-2 m)\left(A m(2 m)^{m-1}\right)^{2 /(1-2 m)}$, and $0<\phi_{2}<\phi_{1}$ when $A<0, \phi_{2}<\phi_{1}<0$ when $A>0$.

Let $M\left(\phi_{e}, 0\right)$ be the coefficient matrix of the linearized system of (13) at equilibrium point $\left(\phi_{e}, 0\right), J\left(\phi_{e}, 0\right)=$ $\operatorname{det}\left(M\left(\phi_{e}, 0\right)\right)$, we have

$$
J\left(\phi_{e}, 0\right)=\frac{m A \phi_{e}\left(\phi_{e}^{2}-c\right)^{m}}{c-\phi_{e}^{2}}-1 .
$$

By the bifurcation theory of dynamical system, we know that $\left(\phi_{e}, 0\right)$ is a saddle point if $J\left(\phi_{e}, 0\right)<0$, a center point if $J\left(\phi_{e}, 0\right)>0$, and a cusp if $J\left(\phi_{e}, 0\right)=0$ and the Poincaré index of $\left(\phi_{e}, 0\right)$ is zero.

By using the properties of equilibrium points and the bifurcation theory of dynamical system, we can show that bifurcation sets and phase portraits of (13) are as drawn in Figure 2.

\section{Existences of Traveling Wave Solutions of (9)}

Liu and Guo [15] investigated the periodic blow-up solutions and their limit forms of a generalized Camassa-Holm equation. We consider existences of periodic blow-up wave solutions and other traveling wave solutions of (9) in this section. Denote that $h_{i}=H\left(\phi_{i}, 0\right), i=0,1,2$, and from Figure 2, we have the following results.

Theorem 1. (i) When $A<0, c>(1-2 m)(A m$ $\left.(2 m)^{m-1}\right)^{2 /(1-2 m)}$, for $h \in\left(h_{1}, h_{2}\right)$, there exists a family of uncountably infinite many smooth periodic wave solutions of (9). Moreover, the smooth periodic wave solutions converge to a solitary wave solution of peak type as $h$ approaches $h_{2}$.

(ii) When $A>0, c>(1-2 m)\left(A m(2 m)^{m-1}\right)^{2 /(1-2 m)}$, for $h \in\left(h_{2}, h_{1}\right)$, there exists a family of uncountably infinite many smooth periodic wave solutions of (9). Moreover, the smooth periodic wave solutions converge to a solitary wave solution of valley type as $h$ approaches $h_{1}$.

Theorem 2. (i) When $A<0, c>(1-2 m)(A m$ $\left.(2 m)^{m-1}\right)^{2 /(1-2 m)}$, for $h \in\left(h_{1}, h_{2}\right)$, there exists a family of uncountably infinite many periodic blow-up wave solutions of (9). Moreover, the periodic blow-up wave solutions converge to a blow-up wave solution as $h$ approaches $h_{2}$.

(ii) When $A>0, c>(1-2 m)\left(A m(2 m)^{m-1}\right)^{2 /(1-2 m)}$, for $h \in\left(h_{2}, h_{1}\right)$, there exists a family of uncountably infinite many periodic blow-up wave solutions of (9). Moreover, the periodic 


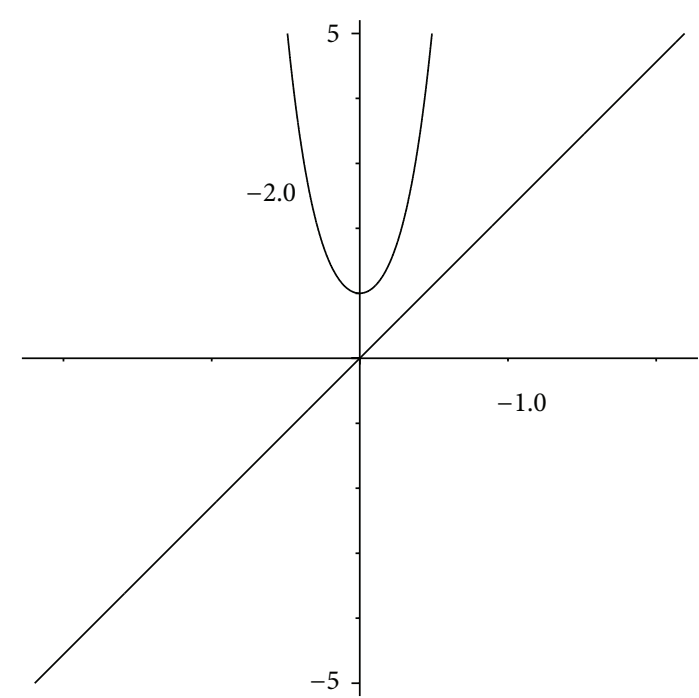

(a) $A<0, c<(1-2 m)\left(A m(2 m)^{m-1}\right)^{2 /(1-2 m)}$

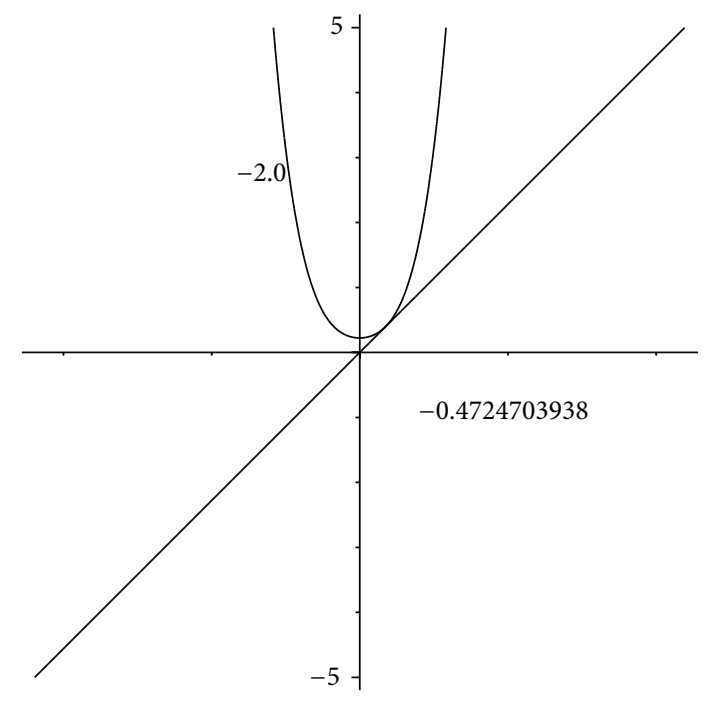

(c) $A<0, c=(1-2 m)\left(A m(2 m)^{m-1}\right)^{2 /(1-2 m)}$

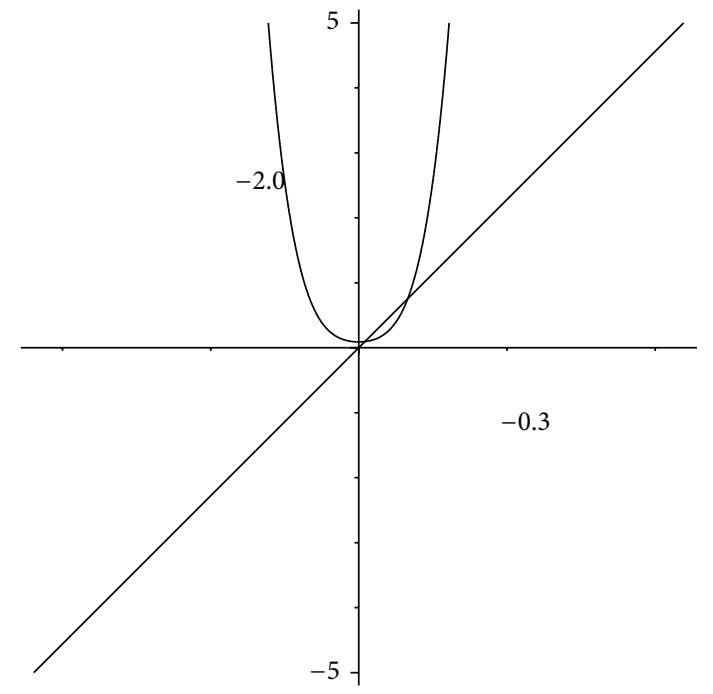

(e) $A<0,(1-2 m)\left(A m(2 m)^{m-1}\right)^{2 /(1-2 m)}<c<0$

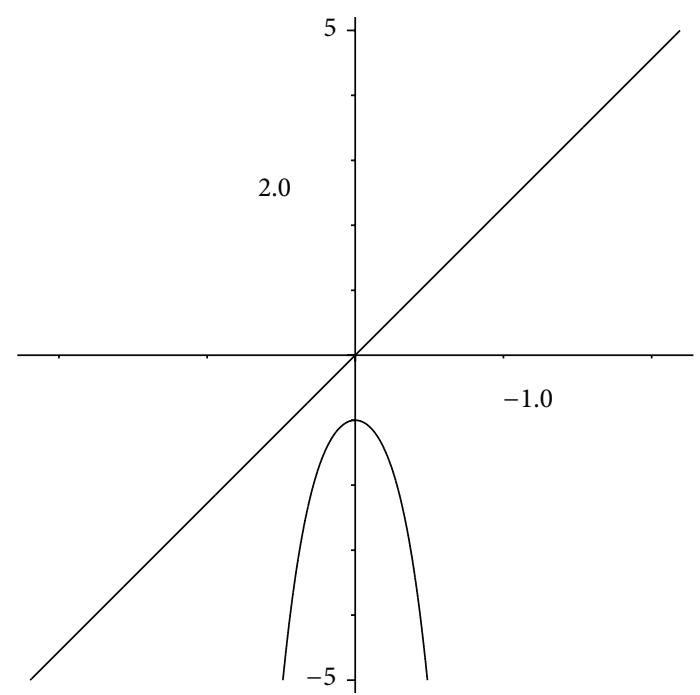

(b) $A>0, c<(1-2 m)\left(A m(2 m)^{m-1}\right)^{2 /(1-2 m)}$

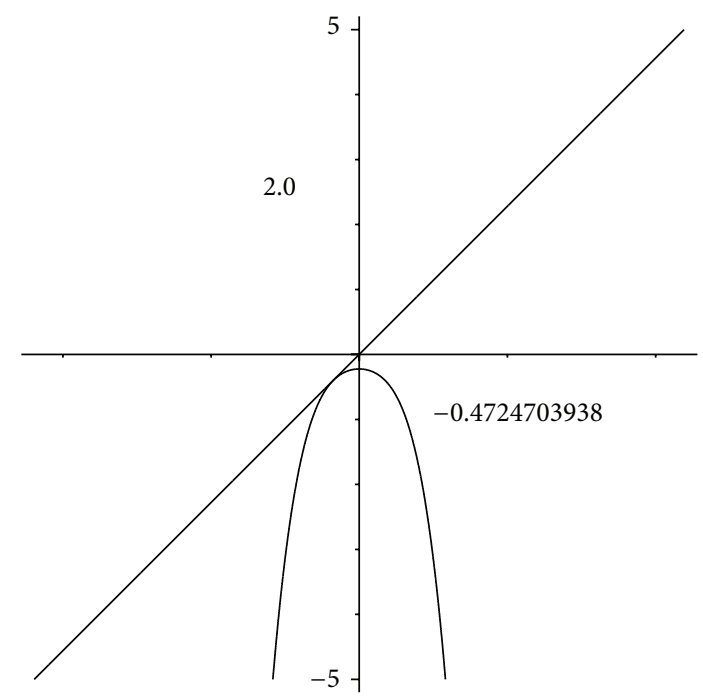

(d) $A>0, c=(1-2 m)\left(A m(2 m)^{m-1}\right)^{2 /(1-2 m)}$

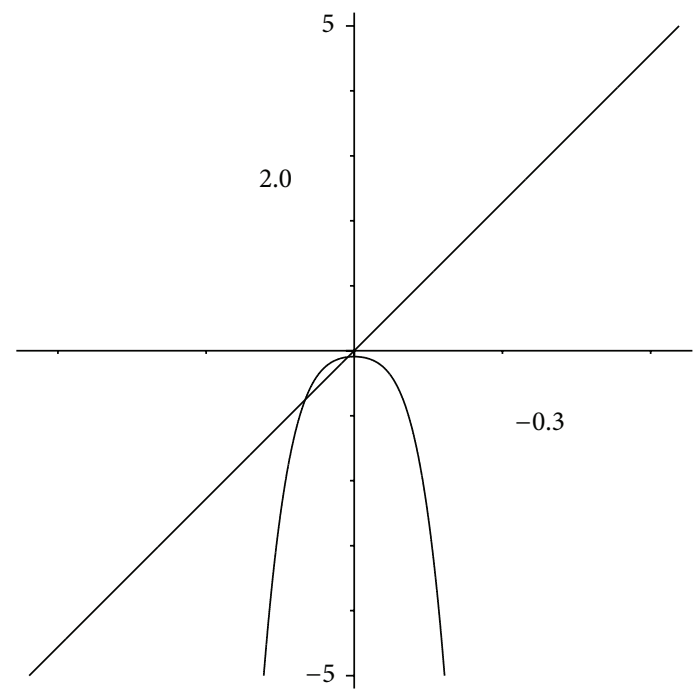

(f) $A>0,(1-2 m)\left(A m(2 m)^{m-1}\right)^{2 /(1-2 m)}<c<0$

FIgure 1: Continued. 


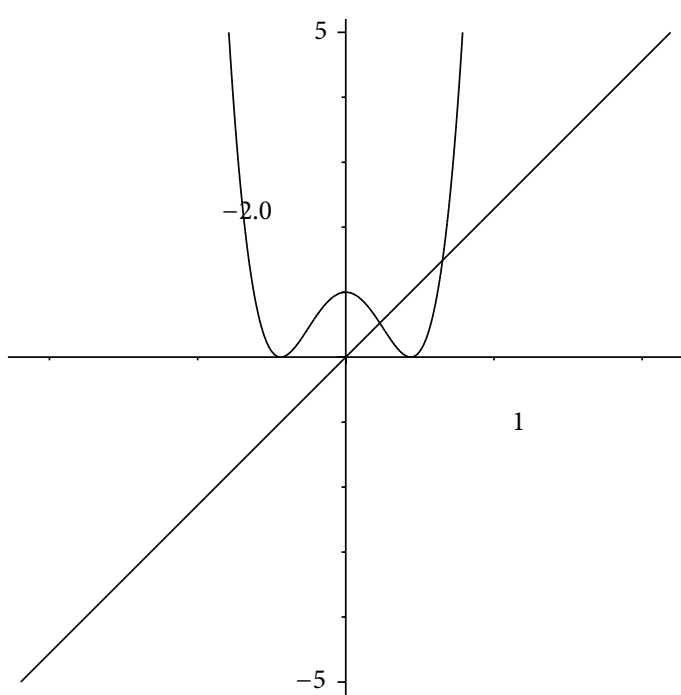

(g) $A<0, c>0$

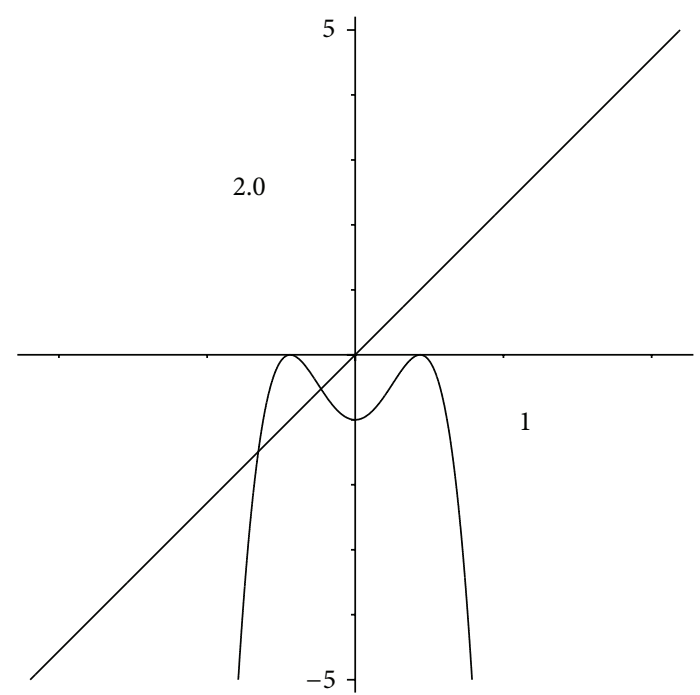

(h) $A>0, c>0$

FIGURE 1: The intersection points of $y=\phi$ and $y=-(1 / 2) A\left(\phi^{2}-c\right)^{m}, m \in Z^{+}$.

blow-up wave solutions converge to a blow-up wave solution as $h$ approaches $h_{1}$.

Theorem 3. When $A \neq 0, c=(1-2 m)\left(A m(2 m)^{m-1}\right)^{2 /(1-2 m)}$, for $h \in\left(-\infty, h_{0}\right)$, there exists a family of uncountably infinite many periodic blow-up wave solutions of (9). Moreover, the periodic blow-up wave solutions converge to a blow-up wave solution as $h$ approaches $h_{0}$.

\section{Explicit Exact Traveling Solutions of (9)}

4.1. Solitary Wave Solutions. From Figure 2(e), we see that there is a homoclinic orbit connecting with the saddle point $\left(\phi_{2}, 0\right)$ and passing point $\left(\phi_{M}, 0\right)$ when $\left.A<0, c\right\rangle(1-$ $2 m)\left(A m(2 m)^{m-1}\right)^{2 /(1-2 m)}$.

When $m=1$, expression of the homoclinic orbit is

$$
y= \pm\left(\phi-\phi_{2}\right) \sqrt{-\frac{1}{3} A\left(\phi_{M}-\phi\right)}, \quad \phi_{2}<\phi \leq \phi_{M}
$$

where $\phi_{2}=\left(-1+\sqrt{1+A^{2} c}\right) / A, \phi_{M}=-\left(1+2 \sqrt{1+A^{2} c}\right) / A$.

Substituting (15) into the $d \phi / d \xi=y$ and integrating it along the homoclinic orbit yields

$$
\int_{\phi}^{\phi_{M}} \frac{d s}{\left(s-\phi_{2}\right) \sqrt{\phi_{M}-s}}=\sqrt{-\frac{1}{3} A}|\xi| .
$$

Completing above integral, we can get a solitary wave solution of peak type of (9) as follows:

$$
u(x, t)=\phi_{M}-\left(\phi_{M}-\phi_{2}\right) \tanh ^{2}\left(\omega_{1}(x-c t)\right)
$$

where $\omega_{1}=(1 / 2) \sqrt{-(1 / 3) A\left(\phi_{M}-\phi_{2}\right)}$.
When $m=2$, expression of the homoclinic orbit is

$$
\begin{array}{r}
y= \pm\left(\phi-\phi_{2}\right) \sqrt{-\frac{1}{5} A\left(\phi_{M}-\phi\right)\left(\left(\phi-b_{1}\right)^{2}+a_{1}^{2}\right)}, \\
\phi_{2}<\phi \leq \phi_{M},
\end{array}
$$

where $\left(\phi-\phi_{2}\right)^{2}\left(-(1 / 5) A\left(\phi_{M}-\phi\right)\left(\left(\phi-b_{1}\right)^{2}+a_{1}^{2}\right)\right)=(1 / 5) A \phi^{5}-$ $(2 / 3) A c \phi^{3}+\phi^{2}+A c \phi+2 h_{2}, h_{2}=-(1 / 10) A \phi_{2}^{5}+(1 / 3) A c \phi_{2}^{3}-$ $(1 / 2) \phi_{2}^{2}-(1 / 2) A c^{2} \phi_{2}$. For example, letting $A=-0.2, c=$ -1.0 , we get that $\phi_{2} \doteq 0.1020955659, \phi_{M} \doteq 2.456246444$, $b_{1} \doteq-1.330218788$, and $a_{1}^{2} \doteq 8.129812158$.

Substituting (18) into the $d \phi / d \xi=y$ and integrating it along the homoclinic orbit yields

$$
\begin{aligned}
& \int_{\phi}^{\phi_{M}} \frac{d s}{\left(s-\phi_{2}\right) \sqrt{\left(\phi_{M}-s\right)\left(\left(s-b_{1}\right)^{2}+a_{1}^{2}\right)}} \\
& \quad=\sqrt{-\frac{1}{5} A}|\xi| .
\end{aligned}
$$

Completing above integral, we can get the implicit representation of the solitary wave solution of peak type of (9) for $u=\phi(x-c t)$ as follows:

$$
\begin{aligned}
& \pm(x-c t) \\
& \quad=\frac{1}{\alpha_{1}\left(\phi_{M}-\phi_{2}-B_{1}\right) \sqrt{-(1 / 5) A B_{1}}}\left\{\mathrm{~F}\left(\varphi_{1}, k_{1}\right)\right. \\
& \left.\quad-\frac{1}{1+\alpha_{1}}\left[\Pi\left(\varphi_{1}, \frac{\alpha_{1}^{2}}{\alpha_{1}^{2}-1}, k_{1}\right)-\alpha_{1} f_{1}\right]\right\},
\end{aligned}
$$

where $\mathrm{F}(\cdot, k), \Pi(\cdot, \cdot, k)$ are the elliptic integrals of the first and third kind, respectively, with the modulus $k[16]$ and $B_{1}=\sqrt{\left(b_{1}-\phi_{M}\right)^{2}+a_{1}^{2}}, \alpha_{1}=\left(\phi_{M}-\phi_{2}+B_{1}\right) /\left(\phi_{M}-\phi_{2}-B_{1}\right)$, 


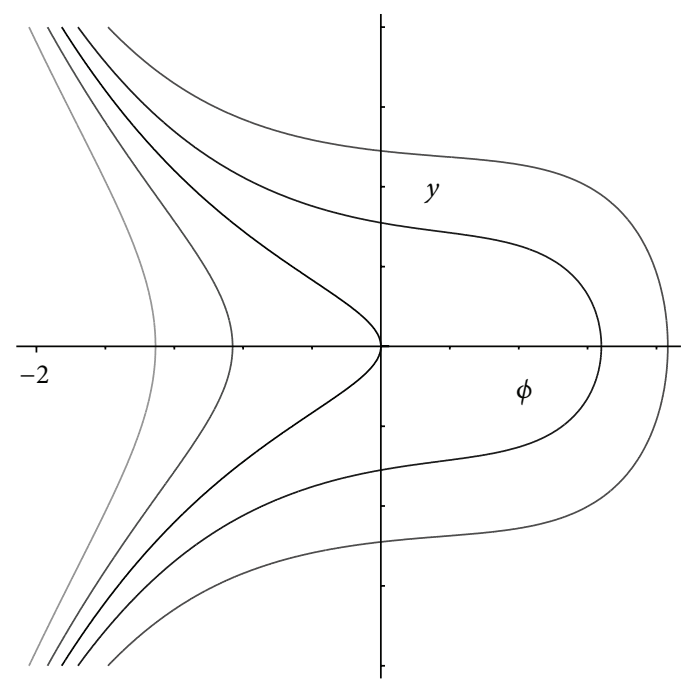

(a) $A<0, c<(1-2 m)\left(A m(2 m)^{m-1}\right)^{2 /(1-2 m)}$

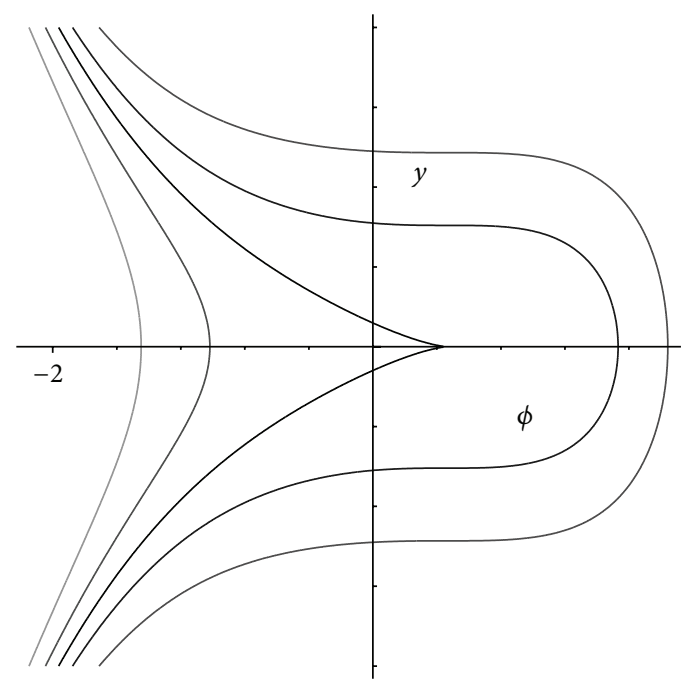

(c) $A<0, c=(1-2 m)\left(A m(2 m)^{m-1}\right)^{2 /(1-2 m)}$

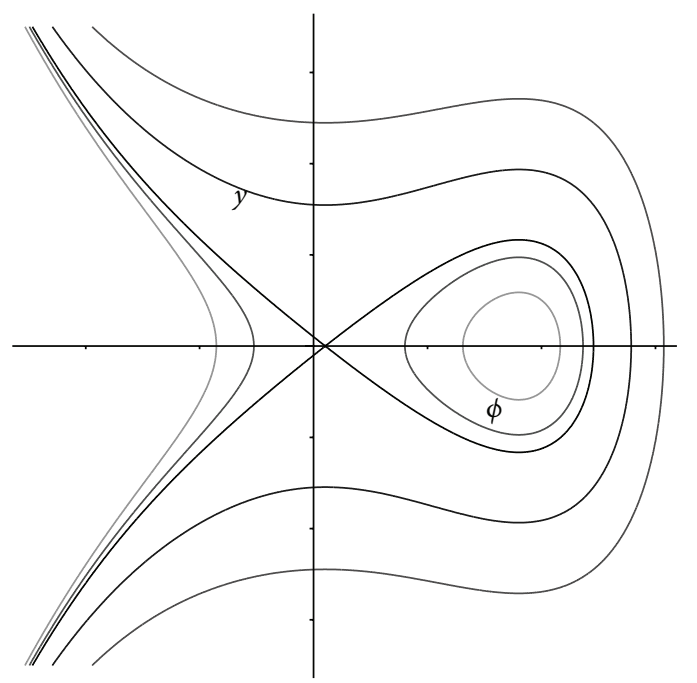

(e) $A<0, c>(1-2 m)\left(A m(2 m)^{m-1}\right)^{2 /(1-2 m)}$

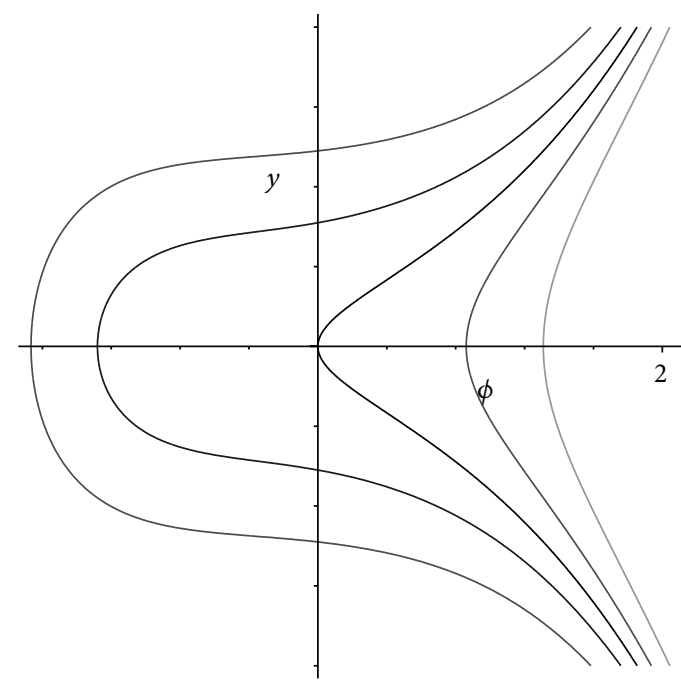

(b) $A>0, c<(1-2 m)\left(A m(2 m)^{m-1}\right)^{2 /(1-2 m)}$

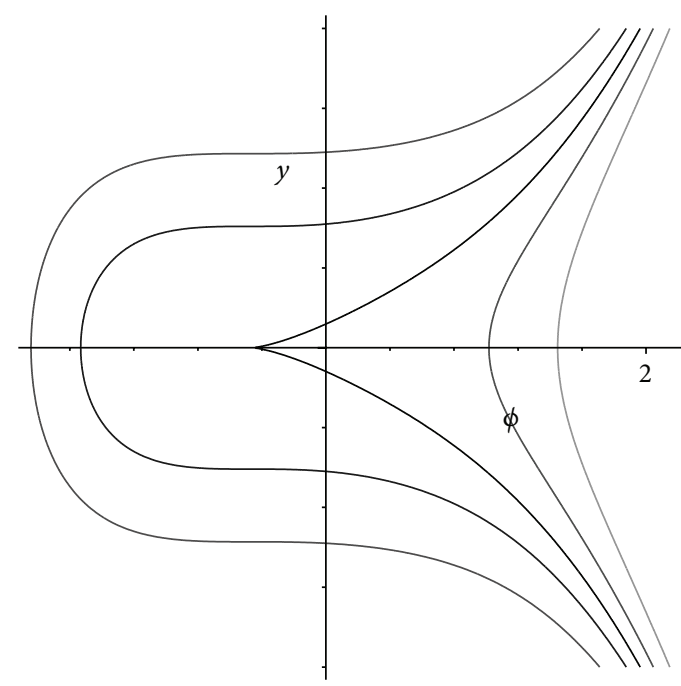

(d) $A>0, c=(1-2 m)\left(A m(2 m)^{m-1}\right)^{2 /(1-2 m)}$

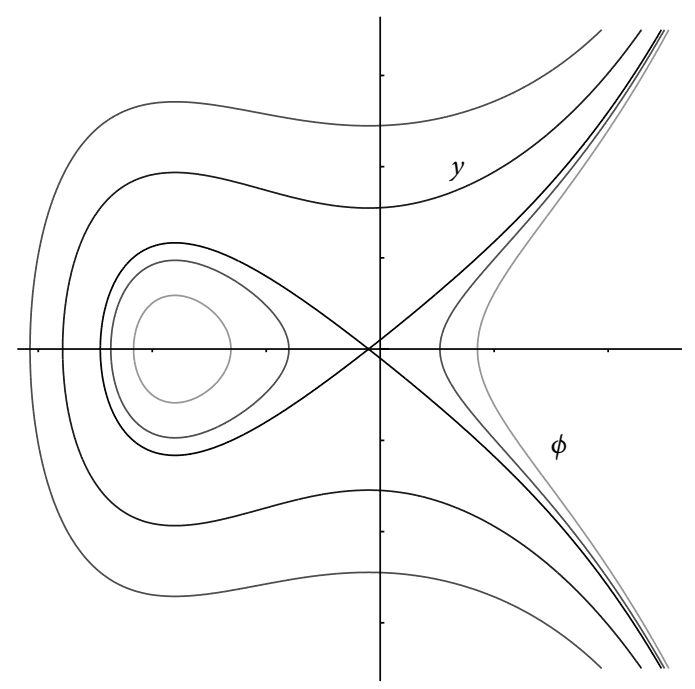

(f) $A>0, c>(1-2 m)\left(A m(2 m)^{m-1}\right)^{2 /(1-2 m)}$

FIgURE 2: Bifurcation sets and phase portraits of system (13). 
$\varphi_{1}=\arccos \left(\left(B_{1}-\phi_{M}+\phi\right) /\left(B_{1}+\phi_{M}-\phi\right)\right)$, $k_{1}=\sqrt{\left(B_{1}-b_{1}+\phi_{M}\right) / 2 B_{1}}, \bar{k}_{1}=\sqrt{1-k_{1}^{2}}$, and $f_{1}=\sqrt{\left(\alpha_{1}^{2}-1\right) /\left(k_{1}^{2}+\bar{k}_{1}^{2} \alpha_{1}^{2}\right)} \ln \mid \sqrt{\left(k_{1}^{2}+\bar{k}_{1}^{2} \alpha_{1}^{2}\right)\left(1-k_{1}^{2} \sin ^{2} \varphi_{1}\right)}+$ $\sqrt{\left(\alpha_{1}^{2}-1\right)\left(1-\cos ^{2} \varphi_{1}\right)} /\left(\sqrt{\left(k_{1}^{2}+\bar{k}_{1}^{2} \alpha_{1}^{2}\right)\left(1-k_{1}^{2} \sin ^{2} \varphi_{1}\right)}\right.$ $\sqrt{\left.\left(\alpha_{1}^{2}-1\right)\left(1-\cos ^{2} \varphi_{1}\right)\right)} \mid$.

From Figure 2(f), we see that there is a homoclinic orbit connecting with the saddle point $\left(\phi_{1}, 0\right)$ and passing point $\left(\phi_{m}, 0\right)$ when $A>0, c>(1-2 m)\left(A m(2 m)^{m-1}\right)^{2 /(1-2 m)}$.

When $m=1$, expression of the homoclinic orbit is

$$
y= \pm\left(\phi_{1}-\phi\right) \sqrt{\frac{1}{3} A\left(\phi-\phi_{m}\right)}, \quad \phi_{m} \leq \phi<\phi_{1},
$$

where $\phi_{1}=\left(-1+\sqrt{1+A^{2} c}\right) / A, \phi_{m}=-\left(1+2 \sqrt{1+A^{2} c}\right) / A$.

Substituting (21) into the $d \phi / d \xi=y$ and integrating it along the homoclinic orbit yields

$$
\int_{\phi_{m}}^{\phi} \frac{d s}{\left(\phi_{1}-s\right) \sqrt{s-\phi_{m}}}=\sqrt{\frac{1}{3} A}|\xi| .
$$

Completing above integral, we can get a solitary wave solution of valley type of (9) as follows:

$$
u(x, t)=\phi_{m}+\left(\phi_{1}-\phi_{m}\right) \tanh ^{2}\left(\omega_{2}(x-c t)\right),
$$

where $\omega_{2}=(1 / 2) \sqrt{(1 / 3) A\left(\phi_{1}-\phi_{m}\right)}$.

When $m=2$, expression of the homoclinic orbit is

$$
\begin{aligned}
& y= \pm\left(\phi_{1}-\phi\right) \sqrt{\frac{1}{5} A\left(\phi-\phi_{m}\right)\left(\left(\phi-b_{2}\right)^{2}+a_{2}^{2}\right)}, \\
& \phi_{m} \leq \phi<\phi_{1},
\end{aligned}
$$

where $\left(\phi_{1}-\phi\right)^{2}\left((1 / 5) A\left(\phi-\phi_{m}\right)\left(\left(\phi-b_{2}\right)^{2}+a_{2}^{2}\right)\right)=(1 / 5) A \phi^{5}-$ $(2 / 3) A c \phi^{3}+\phi^{2}+A c \phi+2 h_{1}, h_{1}=-(1 / 10) A \phi_{1}^{5}+(1 / 3) A c \phi_{1}^{3}-$ $(1 / 2) \phi_{1}^{2}-(1 / 2) A c^{2} \phi_{1}$. For example, letting $A=0.2, c=-1.0$, we get that $\phi_{1} \doteq-0.1020955659, \phi_{m} \doteq-2.456246444, b_{2} \doteq$ 1.330218788 , and $a_{2}^{2} \doteq 8.129812158$.

Substituting (24) into the $d \phi / d \xi=y$ and integrating it along the homoclinic orbit yields

$$
\int_{\phi_{m}}^{\phi} \frac{d s}{\left(\phi_{1}-s\right) \sqrt{\left(s-\phi_{m}\right)\left(\left(s-b_{2}\right)^{2}+a_{2}^{2}\right)}}=\sqrt{\frac{1}{5} A}|\xi| .
$$

Completing above integral, we can get the implicit representation of the solitary wave solution of valley type of (9) for $u=\phi(x-c t)$ as follows:

$$
\begin{aligned}
& \pm(x-c t) \\
& \quad=\frac{1}{\alpha_{2}\left(\phi_{1}-\phi_{m}-B_{2}\right) \sqrt{(1 / 5) A B_{2}}}\left\{\mathrm{~F}\left(\varphi_{2}, k_{2}\right)\right. \\
& \left.\quad-\frac{1}{1+\alpha_{2}}\left[\Pi\left(\varphi_{2}, \frac{\alpha_{2}^{2}}{\alpha_{2}^{2}-1}, k_{2}\right)-\alpha_{2} f_{2}\right]\right\},
\end{aligned}
$$

where $B_{2}=\sqrt{\left(b_{2}-\phi_{m}\right)^{2}+a_{2}^{2}}, \alpha_{2}=\left(\phi_{1}-\phi_{m}+B_{2}\right) /\left(\phi_{1}-\right.$ $\left.\phi_{m}-B_{2}\right), \varphi_{2}=\arccos \left(\left(B_{2}+\phi_{m}-\phi\right) /\left(B_{2}-\phi_{m}+\phi\right)\right)$, $k_{2}=\sqrt{\left(B_{2}+b_{2}-\phi_{m}\right) / 2 B_{2}}, \bar{k}_{2}=\sqrt{1-k_{2}^{2}}$, and $f_{2}=\sqrt{\left(\alpha_{2}^{2}-1\right) /\left(k_{2}^{2}+\bar{k}_{2}^{2} \alpha_{2}^{2}\right)} \ln \mid \sqrt{\left(k_{2}^{2}+\bar{k}_{2}^{2} \alpha_{2}^{2}\right)\left(1-k_{2}^{2} \sin ^{2} \varphi_{2}\right)}+$ $\sqrt{\left(\alpha_{2}^{2}-1\right)\left(1-\cos ^{2} \varphi_{2}\right)} / \sqrt{\left(k_{2}^{2}+\bar{k}_{2}^{2} \alpha_{2}^{2}\right)\left(1-k_{2}^{2} \sin ^{2} \varphi_{2}\right)}$ $\sqrt{\left(\alpha_{2}^{2}-1\right)\left(1-\cos ^{2} \varphi_{2}\right)} \mid$.

4.2. Blow-Up Wave Solutions. From Figure 2(c), we see that there is an open orbit passing the cusp $\left(\phi_{0}, 0\right)$ when $A<0$, $c=(1-2 m)\left(A m(2 m)^{m-1}\right)^{2 /(1-2 m)}$.

When $m=1$, expression of the open orbit is

$$
y= \pm\left(\phi_{0}-\phi\right) \sqrt{-\frac{1}{3} A\left(\phi_{0}-\phi\right)}, \quad-\infty<\phi \leq \phi_{0},
$$

where $\phi_{0}=-1 / A$.

Substituting (27) into the $d \phi / d \xi=y$ and integrating it along the open orbit yields

$$
\int_{-\infty}^{\phi} \frac{d s}{\left(\phi_{0}-s\right) \sqrt{\phi_{0}-s}}=\sqrt{-\frac{1}{3} A}|\xi| .
$$

Completing above integral, we can get a blow-up wave solution of (9) as follows:

$$
u(x, t)=\phi_{0}-\frac{1}{\left(\omega_{3}\left(x+\left(1 / A^{2}\right) t\right)\right)^{2}},
$$

where $\omega_{3}=(1 / 2) \sqrt{-(1 / 3) A}$.

When $m=2$, expression of the open orbit is

$$
\begin{aligned}
& y= \pm\left(\phi_{0}-\phi\right) \sqrt{-\frac{1}{5} A\left(\phi_{0}-\phi\right)\left(\left(\phi-b_{3}\right)^{2}+a_{3}^{2}\right)}, \\
&-\infty<\phi \leq \phi_{0},
\end{aligned}
$$

where $\left(\phi_{0}-\phi\right)^{2}\left(-(1 / 5) A\left(\phi_{0}-\phi\right)\left(\left(\phi-b_{3}\right)^{2}+a_{3}^{2}\right)\right)=(1 / 5) A \phi^{5}-$ $(2 / 3) A c \phi^{3}+\phi^{2}+A c \phi+2 h_{0}, h_{0}=-(1 / 10) A \phi_{0}^{5}+(1 / 3) A c \phi_{0}^{3}-$ $(1 / 2) \phi_{0}^{2}-(1 / 2) A c^{2} \phi_{0}$. For example, letting $A=-1.2, c \doteq$ -0.6641616057 , we get that $\phi_{0} \doteq 0.4705128788, b_{3} \doteq$ -0.7057693180 , and $a_{3}^{2} \doteq 3.044055903$.

Substituting (30) into the $d \phi / d \xi=y$ and integrating it along the open orbit yields

$$
\int_{-\infty}^{\phi} \frac{d s}{\left(\phi_{0}-s\right) \sqrt{\left(\phi_{0}-s\right)\left(\left(s-b_{3}\right)^{2}+a_{3}^{2}\right)}}=\sqrt{-\frac{1}{5} A}|\xi| .
$$

Completing above integral, we can get the implicit representation of the blow-up wave solution of (9) for $u=\phi(x+$ $\left.(3 / 4) \sqrt[3]{1 / A^{2}} t\right)$ as follows:

$$
\begin{aligned}
& \pm\left(x+\frac{3}{4} \sqrt[3]{\frac{1}{A^{2}} t}\right)=\frac{1}{B_{3} \sqrt{-(1 / 5) A B_{3}}}\left\{\mathrm{~F}\left(\varphi_{3}, k_{3}\right)\right. \\
& \left.-2 \mathrm{E}\left(\varphi_{3}, k_{3}\right)+\frac{2 \sin \varphi_{3} \sqrt{1-k_{3}^{2} \sin ^{2} \varphi_{3}}}{1+\cos \varphi_{3}}\right\},
\end{aligned}
$$


where $\mathrm{E}(\cdot, k)$ is the elliptic integral of the second kind with the modulus $k[16]$ and $B_{3}=\sqrt{\left(b_{3}-\phi_{0}\right)^{2}+a_{3}^{2}}, \varphi_{3}=\arccos \left(\left(\phi_{0}-\right.\right.$ $\left.\left.B_{3}-\phi\right) /\left(\phi_{0}+B_{3}-\phi\right)\right), k_{3}=\sqrt{\left(B_{3}-b_{3}+\phi_{0}\right) / 2 B_{3}}$.

From Figure 2(d), we see that there is an open orbit passing the cusp $\left(\phi_{0}, 0\right)$ when $A>0, c=(1-$ $2 m)\left(A m(2 m)^{m-1}\right)^{2 /(1-2 m)}$.

When $m=1$, expression of the open orbit is

$$
y= \pm\left(\phi-\phi_{0}\right) \sqrt{\frac{1}{3} A\left(\phi-\phi_{0}\right)}, \quad \phi_{0} \leq \phi<+\infty,
$$

where $\phi_{0}=-1 / A$.

Substituting (33) into the $d \phi / d \xi=y$ and integrating it along the open orbit yields

$$
\int_{\phi}^{+\infty} \frac{d s}{\left(s-\phi_{0}\right) \sqrt{s-\phi_{0}}}=\sqrt{\frac{1}{3} A}|\xi| .
$$

Completing above integral, we can get a blow-up wave solution of (9) as follows:

$$
u(x, t)=\phi_{0}+\frac{1}{\left(\omega_{4}\left(x+\left(1 / A^{2}\right) t\right)\right)^{2}},
$$

where $\omega_{4}=(1 / 2) \sqrt{(1 / 3) A}$.

When $m=2$, expression of the open orbit is

$$
\begin{aligned}
& y= \pm\left(\phi-\phi_{0}\right) \sqrt{\frac{1}{5} A\left(\phi-\phi_{0}\right)\left(\left(\phi-b_{4}\right)^{2}+a_{4}^{2}\right)} \\
& \phi_{0} \leq \phi<+\infty,
\end{aligned}
$$

where $\left(\phi-\phi_{0}\right)^{2}\left((1 / 5) A\left(\phi-\phi_{0}\right)\left(\left(\phi-b_{4}\right)^{2}+a_{4}^{2}\right)\right)=(1 / 5) A \phi^{5}-$ $(2 / 3) A c \phi^{3}+\phi^{2}+A c \phi+2 h_{0}, h_{0}=-(1 / 10) A \phi_{0}^{5}+(1 / 3) A c \phi_{0}^{3}-$ $(1 / 2) \phi_{0}^{2}-(1 / 2) A c^{2} \phi_{0}$. For example, letting $A=1.2, c \doteq$ -0.6641616057 , we get that $\phi_{0} \doteq-0.4705128788, b_{4} \doteq$ -0.7057693180 , and $a_{4}^{2} \doteq 3.044055903$.

Substituting (36) into the $d \phi / d \xi=y$ and integrating it along the open orbit yields

$$
\int_{\phi}^{+\infty} \frac{d s}{\left(s-\phi_{0}\right) \sqrt{\left(s-\phi_{0}\right)\left(\left(s-b_{4}\right)^{2}+a_{4}^{2}\right)}}=\sqrt{\frac{1}{5} A}|\xi| .
$$

Completing above integral, we can get the implicit representation of the blow-up wave solution of (9) for $u=\phi(x+$ $\left.(3 / 4) \sqrt[3]{\left(1 / A^{2}\right)} t\right)$ as follows:

$$
\begin{gathered}
\pm\left(x+\frac{3}{4} \sqrt[3]{\frac{1}{A^{2}}} t\right)=\frac{1}{B_{4} \sqrt{(1 / 5) A B_{4}}}\left\{\mathrm{~F}\left(\varphi_{4}, k_{4}\right)\right. \\
\left.-2 \mathrm{E}\left(\varphi_{4}, k_{4}\right)+\frac{2 \sin \varphi_{4} \sqrt{1-k_{4}^{2} \sin ^{2} \varphi_{4}}}{1+\cos \varphi_{4}}\right\},
\end{gathered}
$$

where $B_{4}=\sqrt{\left(b_{4}-\phi_{0}\right)^{2}+a_{4}^{2}}, \varphi_{4}=\arccos \left(\left(\phi-\phi_{0}-B_{4}\right) /(\phi-\right.$ $\left.\left.\phi_{0}+B_{4}\right)\right)$, and $k_{4}=\sqrt{\left(B_{4}+b_{4}-\phi_{0}\right) / 2 B_{4}}$.
From Figure 2(e), we see that there is an open orbit passing the saddle point $\left(\phi_{2}, 0\right)$ when $\left.A<0, c\right\rangle$ (1$2 m)\left(A m(2 m)^{m-1}\right)^{2 /(1-2 m)}$.

When $m=1$, expression of the open orbit is

$$
y= \pm\left(\phi_{2}-\phi\right) \sqrt{-\frac{1}{3} A\left(\phi_{M}-\phi\right)}, \quad-\infty<\phi \leq \phi_{2},
$$

where $\phi_{2}, \phi_{M}$ are given in (15).

Substituting (39) into the $d \phi / d \xi=y$ and integrating it along the open orbit yields

$$
\int_{-\infty}^{\phi} \frac{d s}{\left(\phi_{2}-s\right) \sqrt{\phi_{M}-s}}=\sqrt{-\frac{1}{3} A}|\xi| .
$$

Completing above integral, we can get a blow-up wave solution of (9) as follows:

$$
u(x, t)=\phi_{M}-\left(\phi_{M}-\phi_{2}\right) \operatorname{coth}^{2}\left(\omega_{1}(x-c t)\right),
$$

where $\omega_{1}$ is given in (17).

When $m=2$, expression of the open orbit is

$$
\begin{array}{r}
y= \pm\left(\phi_{2}-\phi\right) \sqrt{-\frac{1}{5} A\left(\phi_{M}-\phi\right)\left(\left(\phi-b_{1}\right)^{2}+a_{1}^{2}\right)}, \\
-\infty<\phi \leq \phi_{2},
\end{array}
$$

where $\phi_{2}, \phi_{M}, b_{1}$, and $a_{1}^{2}$ are given in (18).

Substituting (42) into the $d \phi / d \xi=y$ and integrating it along the open orbit yields

$$
\begin{aligned}
& \int_{-\infty}^{\phi} \frac{d s}{\left(\phi_{2}-s\right) \sqrt{\left(\phi_{M}-s\right)\left(\left(s-b_{1}\right)^{2}+a_{1}^{2}\right)}} \\
& \quad=\sqrt{-\frac{1}{5} A}|\xi| .
\end{aligned}
$$

Completing above integral, we can get the implicit representation of the blow-up wave solution of (9) for $u=\phi(x-c t)$ as follows:

$$
\begin{aligned}
& \pm(x-c t) \\
& \quad=\frac{1}{\alpha_{1}\left(\phi_{2}-\phi_{M}+B_{1}\right) \sqrt{-(1 / 5) A B_{1}}}\left\{\mathrm{~F}\left(\bar{\varphi}_{1}, k_{1}\right)\right. \\
& \left.\quad-\frac{1}{1+\alpha_{1}}\left[\Pi\left(\bar{\varphi}_{1}, \frac{\alpha_{1}^{2}}{\alpha_{1}^{2}-1}, k_{1}\right)+\alpha_{1} f_{1}\right]\right\},
\end{aligned}
$$

where $\bar{\varphi}_{1}=\pi-\varphi_{1}$ and $B_{1}, \alpha_{1}, \varphi_{1}, k_{1}$, and $f_{1}$ are given in (20).

From Figure 2(f), we see that there is an open orbit passing the saddle point $\left(\phi_{1}, 0\right)$ when $A>0, c>(1-$ $2 m)\left(A m(2 m)^{m-1}\right)^{2 /(1-2 m)}$.

When $m=1$, expression of the open orbit is

$$
y= \pm\left(\phi-\phi_{1}\right) \sqrt{\frac{1}{3} A\left(\phi-\phi_{m}\right)}, \quad \phi_{1} \leq \phi<+\infty
$$

where $\phi_{1}, \phi_{m}$ are given in (21). 
Substituting (45) into the $d \phi / d \xi=y$ and integrating it along the open orbit yields

$$
\int_{\phi}^{+\infty} \frac{d s}{\left(s-\phi_{1}\right) \sqrt{s-\phi_{m}}}=\sqrt{\frac{1}{3} A}|\xi| .
$$

Completing above integral, we can get a blow-up wave solution of (9) as follows:

$$
u(x, t)=\phi_{m}+\left(\phi_{1}-\phi_{m}\right) \operatorname{coth}^{2}\left(\omega_{2}(x-c t)\right),
$$

where $\omega_{2}$ is given in (23).

When $m=2$, expression of the open orbit is

$$
\begin{aligned}
& y= \pm\left(\phi-\phi_{1}\right) \sqrt{\frac{1}{5} A\left(\phi-\phi_{m}\right)\left(\left(\phi-b_{2}\right)^{2}+a_{2}^{2}\right)}, \\
& \phi_{1} \leq \phi<+\infty,
\end{aligned}
$$

where $\phi_{1}, \phi_{m}, b_{2}$, and $a_{2}^{2}$ are given in (24).

Substituting (48) into the $d \phi / d \xi=y$ and integrating it along the open orbit yields

$$
\int_{\phi}^{+\infty} \frac{d s}{\left(s-\phi_{1}\right) \sqrt{\left(s-\phi_{m}\right)\left(\left(s-b_{2}\right)^{2}+a_{2}^{2}\right)}}=\sqrt{\frac{1}{5} A}|\xi| .
$$

Completing above integral, we can get the implicit representation of the blow-up wave solution of (9) for $u=\phi(x-c t)$ as follows:

$$
\begin{aligned}
& \pm(x-c t) \\
& \quad=\frac{1}{\alpha_{2}\left(B_{2}+\phi_{m}-\phi_{1}\right) \sqrt{(1 / 5) A B_{2}}}\left\{\mathrm{~F}\left(\bar{\varphi}_{2}, k_{2}\right)\right. \\
& \left.\quad-\frac{1}{1+\alpha_{2}}\left[\Pi\left(\bar{\varphi}_{2}, \frac{\alpha_{2}^{2}}{\alpha_{2}^{2}-1}, k_{2}\right)+\alpha_{2} f_{2}\right]\right\},
\end{aligned}
$$

where $\bar{\varphi}_{2}=\pi-\varphi_{2}$ and $B_{2}, \alpha_{2}, \varphi_{2}, k_{2}$, and $f_{2}$ are given in (26).

4.3. Smooth Periodic Wave Solutions. From Figure 2(e), we see that there is a periodic orbit passing points $\left(\gamma_{1}, 0\right)$ and $\left(\gamma_{2}, 0\right)$ when $\left.A<0, c\right\rangle(1-2 m)\left(A m(2 m)^{m-1}\right)^{2 /(1-2 m)}$, $h \in\left(h_{1}, h_{2}\right)$.

When $m=1$, expression of the periodic orbit is

$$
y= \pm \sqrt{-\frac{1}{3} A\left(\gamma_{1}-\phi\right)\left(\phi-\gamma_{2}\right)\left(\phi-\gamma_{3}\right)},
$$

$$
\gamma_{2} \leq \phi \leq \gamma_{1},
$$

where $\gamma_{1}, \gamma_{2}$, and $\gamma_{3}\left(\gamma_{3}<0<\gamma_{2}<\gamma_{1}\right)$ are three real roots of $(1 / 3) A \psi^{3}+\psi^{2}-A c \psi+2 h=0$. For example, taking $A=-1.5$, $c=1.2$, we have $h_{1} \doteq-1.802532163, h_{2} \doteq 0.3062358669$. Letting $h=-0.2$, we get that $\gamma_{1} \doteq 3.083397695, \gamma_{2} \doteq$ 0.2018680190 , and $\gamma_{3} \doteq-1.285265714$.

Substituting (51) into the $d \phi / d \xi=y$ and integrating it along the periodic orbit yields

$$
\int_{\gamma_{2}}^{\phi} \frac{d s}{\sqrt{\left(\gamma_{1}-s\right)\left(s-\gamma_{2}\right)\left(s-\gamma_{3}\right)}}=\sqrt{-\frac{1}{3} A}|\xi| .
$$

Completing above integral, we can get a smooth periodic wave solution of (9) as follows:

$$
\begin{aligned}
& u(x, t) \\
& =\frac{\gamma_{2}\left(\gamma_{3}-\gamma_{1}\right)+\gamma_{3}\left(\gamma_{1}-\gamma_{2}\right) \operatorname{sn}^{2}\left(\omega_{5}(x-c t), k_{5}\right)}{\left(\gamma_{3}-\gamma_{1}\right)+\left(\gamma_{1}-\gamma_{2}\right) \operatorname{sn}^{2}\left(\omega_{5}(x-c t), k_{5}\right)},
\end{aligned}
$$

where $\operatorname{sn}(\cdot, k)$ is the Jacobian elliptic function with the modulus $k$ [16], and $\omega_{5}=(1 / 2) \sqrt{-(1 / 3) A\left(\gamma_{1}-\gamma_{3}\right)}, k_{5}=$ $\sqrt{\left(\gamma_{1}-\gamma_{2}\right) /\left(\gamma_{1}-\gamma_{3}\right)}$.

From Figure 2(f), we see that there is a periodic orbit passing points $\left(\gamma_{2}, 0\right)$ and $\left(\gamma_{3}, 0\right)$ when $A>0, c>(1-$ $2 m)\left(A m(2 m)^{m-1}\right)^{2 /(1-2 m)}, h \in\left(h_{2}, h_{1}\right)$.

When $m=1$, expression of the periodic orbit is

$$
y= \pm \sqrt{\frac{1}{3} A\left(\gamma_{1}-\phi\right)\left(\gamma_{2}-\phi\right)\left(\phi-\gamma_{3}\right)}, \quad \gamma_{3} \leq \phi \leq \gamma_{2},
$$

where $\gamma_{1}, \gamma_{2}$, and $\gamma_{3}\left(\gamma_{3}<\gamma_{2}<0<\gamma_{1}\right)$ are three real roots of $(1 / 3) A \psi^{3}+\psi^{2}-A c \psi+2 h=0$. For example, taking $A=1.5, c=$ 1.2 , we have $h_{1} \doteq 0.3062358669, h_{2} \doteq-1.802532163$. Letting $h=-1.3$, we get that $\gamma_{1} \doteq 1.751240424, \gamma_{2} \doteq-1.134926411$, and $\gamma_{3} \doteq-2.616314014$.

Substituting (54) into the $d \phi / d \xi=y$ and integrating it along the periodic orbit yields

$$
\int_{\phi}^{\gamma_{2}} \frac{d s}{\sqrt{\left(\gamma_{1}-s\right)\left(\gamma_{2}-s\right)\left(s-\gamma_{3}\right)}}=\sqrt{\frac{1}{3} A}|\xi| .
$$

Completing above integral, we can get a smooth periodic wave solution of (9) as follows:

$$
\begin{aligned}
& u(x, t) \\
& =\frac{\gamma_{2}\left(\gamma_{3}-\gamma_{1}\right)+\gamma_{1}\left(\gamma_{2}-\gamma_{3}\right) \operatorname{sn}^{2}\left(\omega_{6}(x-c t), k_{6}\right)}{\left(\gamma_{3}-\gamma_{1}\right)+\left(\gamma_{2}-\gamma_{3}\right) \operatorname{sn}^{2}\left(\omega_{6}(x-c t), k_{6}\right)},
\end{aligned}
$$

where $\omega_{6}=$

$(1 / 2) \sqrt{(1 / 3) A\left(\gamma_{1}-\gamma_{3}\right)}, \quad k_{6}=$ $\sqrt{\left(\gamma_{2}-\gamma_{3}\right) /\left(\gamma_{1}-\gamma_{3}\right)}$.

4.4. Periodic Blow-Up Wave Solutions. From Figure 2(e), we see that there is an open orbit passing point $\left(\gamma_{3}, 0\right)$ when $A<$ $0, c>(1-2 m)\left(A m(2 m)^{m-1}\right)^{2 /(1-2 m)}, h \in\left(h_{1}, h_{2}\right)$.

When $m=1$, expression of the open orbit is

$$
y= \pm \sqrt{-\frac{1}{3} A\left(\gamma_{1}-\phi\right)\left(\gamma_{2}-\phi\right)\left(\gamma_{3}-\phi\right)}
$$

$$
-\infty<\phi \leq \gamma_{3},
$$

where $\gamma_{1}, \gamma_{2}$, and $\gamma_{3}$ are given in (51).

Substituting (57) into the $d \phi / d \xi=y$ and integrating it along the open orbit yields

$$
\int_{-\infty}^{\phi} \frac{d s}{\sqrt{\left(\gamma_{1}-s\right)\left(\gamma_{2}-s\right)\left(\gamma_{3}-s\right)}}=\sqrt{-\frac{1}{3} A}|\xi| .
$$


Completing above integral, we can get a periodic blow-up wave solution of (9) as follows:

$$
u(x, t)=\gamma_{1}-\left(\gamma_{1}-\gamma_{3}\right) \mathrm{ns}^{2}\left(\omega_{5}(x-c t), k_{5}\right),
$$

where $\mathrm{ns}(\cdot, k)$ is the Jacobian elliptic function with the modulus $k$ [16], and $\omega_{5}, k_{5}$ are given in (53).

From Figure 2(f), we see that there is an open orbit passing point $\left(\gamma_{1}, 0\right)$ when $A>0, c>(1-$ $2 m)\left(A m(2 m)^{m-1}\right)^{2 /(1-2 m)}, h \in\left(h_{2}, h_{1}\right)$.

When $m=1$, expression of the periodic orbit is

$$
y= \pm \sqrt{\frac{1}{3} A\left(\phi-\gamma_{1}\right)\left(\phi-\gamma_{2}\right)\left(\phi-\gamma_{3}\right)},
$$

$$
\gamma_{1} \leq \phi<+\infty
$$

where $\gamma_{1}, \gamma_{2}$, and $\gamma_{3}$ are given in (54).

Substituting (60) into the $d \phi / d \xi=y$ and integrating it along the open orbit yields

$$
\int_{\phi}^{+\infty} \frac{d s}{\sqrt{\left(s-\gamma_{1}\right)\left(s-\gamma_{2}\right)\left(s-\gamma_{3}\right)}}=\sqrt{\frac{1}{3} A}|\xi| .
$$

Completing above integral, we can get a periodic blow-up wave solution of (9) as follows:

$$
u(x, t)=\gamma_{3}+\left(\gamma_{1}-\gamma_{3}\right) \mathrm{ns}^{2}\left(\omega_{6}(x-c t), k_{6}\right),
$$

where $\omega_{6}, k_{6}$ are given in (56).

\section{Conclusion}

In this paper, the modified Novikov equation is studied by using the bifurcation theory of dynamical system and the method of phase portraits analysis. For a special case, the existences of different kinds of nonlinear waves and limit forms of two periodic wave solutions of the equation are stated in Theorems 1-3. All possible exact parametric representations of the nonlinear waves also are presented in (17), (20), (23), and (29) and so forth. The previous results of the equation are enriched.

\section{Conflict of Interests}

The authors declare that there is no conflict of interests regarding the publication of this paper.

\section{Acknowledgments}

This work is supported by the National Natural Science Foundation of China under Grant no. 11461022 and the Natural Science Foundations of Yunnan Province, China, under Grant nos. 2014FA037, 2013 FZ117.

\section{References}

[1] V. Novikov, "Generalizations of the Camassa-Holm equation," Journal of Physics A: Mathematical and Theoretical, vol. 42, no. 34, Article ID 342002, 14 pages, 2009.
[2] A. N. Hone, H. Lundmark, and J. Szmigielski, "Explicit multipeakon solutions of Novikov's cubically nonlinear integrable Camassa-Holm type equation," Dynamics of Partial Differential Equations, vol. 6, no. 3, pp. 253-289, 2009.

[3] A. N. Hone and J. P. Wang, "Integrable peakon equations with cubic nonlinearity," Journal of Physics A. Mathematical and Theoretical, vol. 41, no. 37, Article ID 372002, 10 pages, 2008.

[4] Z. Jiang and L. Ni, "Blow-up phenomenon for the integrable Novikov equation," Journal of Mathematical Analysis and Applications, vol. 385, no. 1, pp. 551-558, 2012.

[5] X. L. Wu and Z. Y. Yin, "Global weak solutions for the Novikov equation," Journal of Physics A: Mathematical and Theoretical, vol. 44, no. 5, Article ID 055202, 17 pages, 2011.

[6] Y. Bozhkov, I. L. Freire, and N. H. Ibragimov, "Group analysis of the Novikov equation," Computational \& Applied Mathematics, vol. 33, no. 1, pp. 193-202, 2014.

[7] J. Li, "Exact cuspon and compactons of the Novikov equation," International Journal of Bifurcation and Chaos, vol. 24, no. 3, Article ID 1450037, 8 pages, 2014.

[8] A. A. Himonas and C. Holliman, "The Cauchy problem for the Novikov equation,” Nonlinearity, vol. 25, no. 2, pp. 449-479, 2012.

[9] K. Grayshan, "Peakon solutions of the Novikov equation and properties of the data-to-solution map," Journal of Mathematical Analysis and Applications, vol. 397, no. 2, pp. 515-521, 2013.

[10] S. Y. Lai and M. Wu, "The local strong and weak solutions to a generalized Novikov equation,” Boundary Value Problems, vol. 2013, article 134, 2013.

[11] S. Lai, H. Yan, and N. Li, "The global solution and blow-up phenomena to a modified Novikov equation," Boundary Value Problems, vol. 2014, article 16, 9 pages, 2014.

[12] S. M. Zhou and R. Chen, "A fewremarks on the generalized Novikov equation," Journal of Inequalities and Applications, vol. 2013, article 560, 2013.

[13] Y. S. Mi and C. L. Mu, "On the Cauchy problem for the modified Novikov equation with peakon solutions," Journal of Differential Equations, vol. 254, no. 3, pp. 961-982, 2013.

[14] J. B. Li and H. H. Dai, On the Study of Singular Nonlinear Traveling Wave Equation: Dynamical System Approach, Science Press, Beijing, China, 2007.

[15] Z. Liu and B. L. Guo, "Periodic blow-up solutions and their limit forms for the generalized Camassa-Holm equation," Progress in Natural Science, vol. 18, no. 3, pp. 259-266, 2008.

[16] P. F. Byrd and M. D. Friedman, Handbook of Elliptic Integrals for Engineers and Physicists, Springer, Berlin, Germany, 1971. 


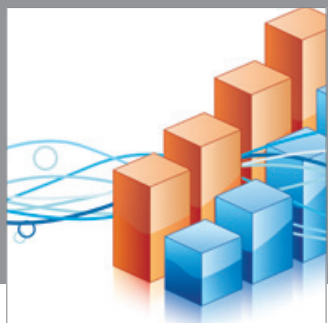

Advances in

Operations Research

mansans

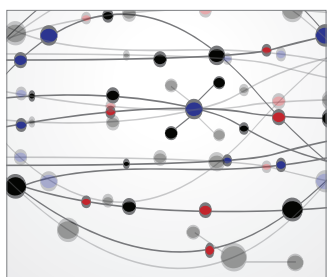

The Scientific World Journal
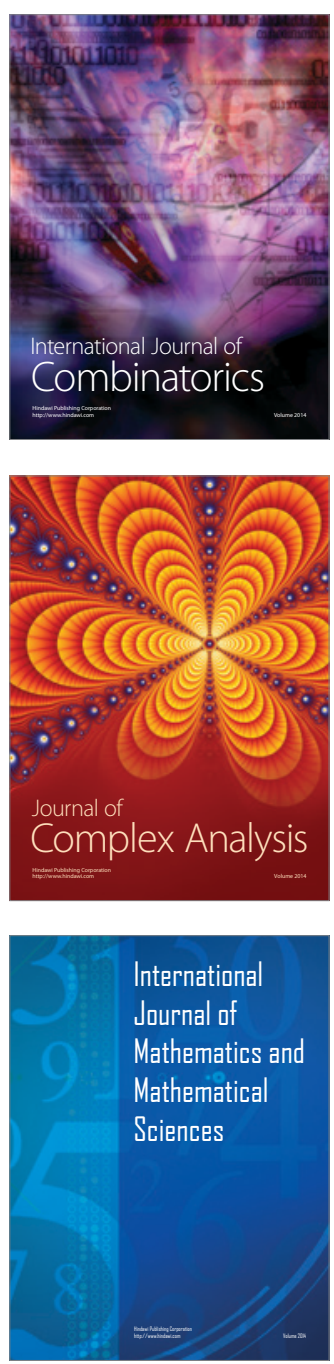
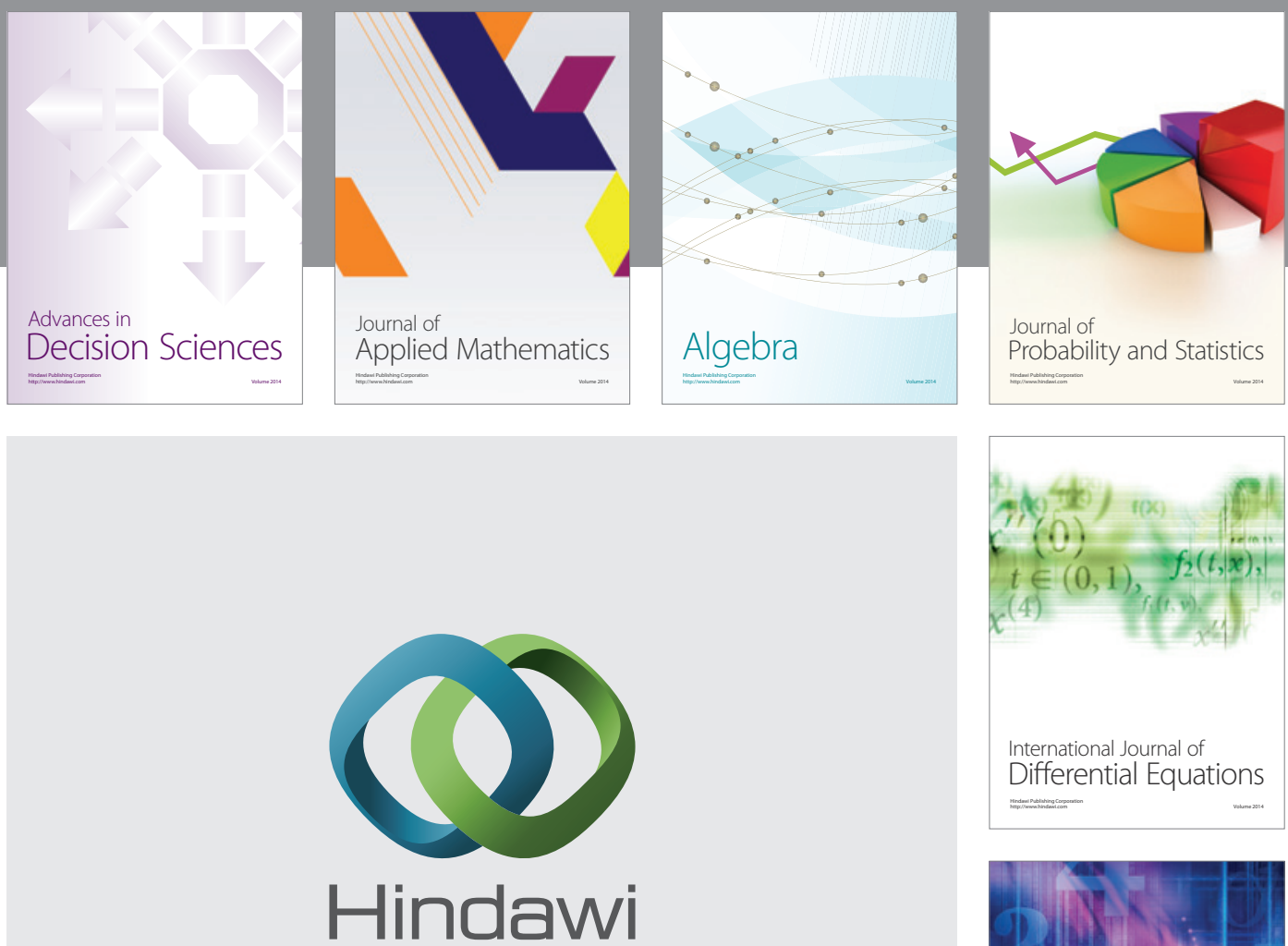

Submit your manuscripts at http://www.hindawi.com
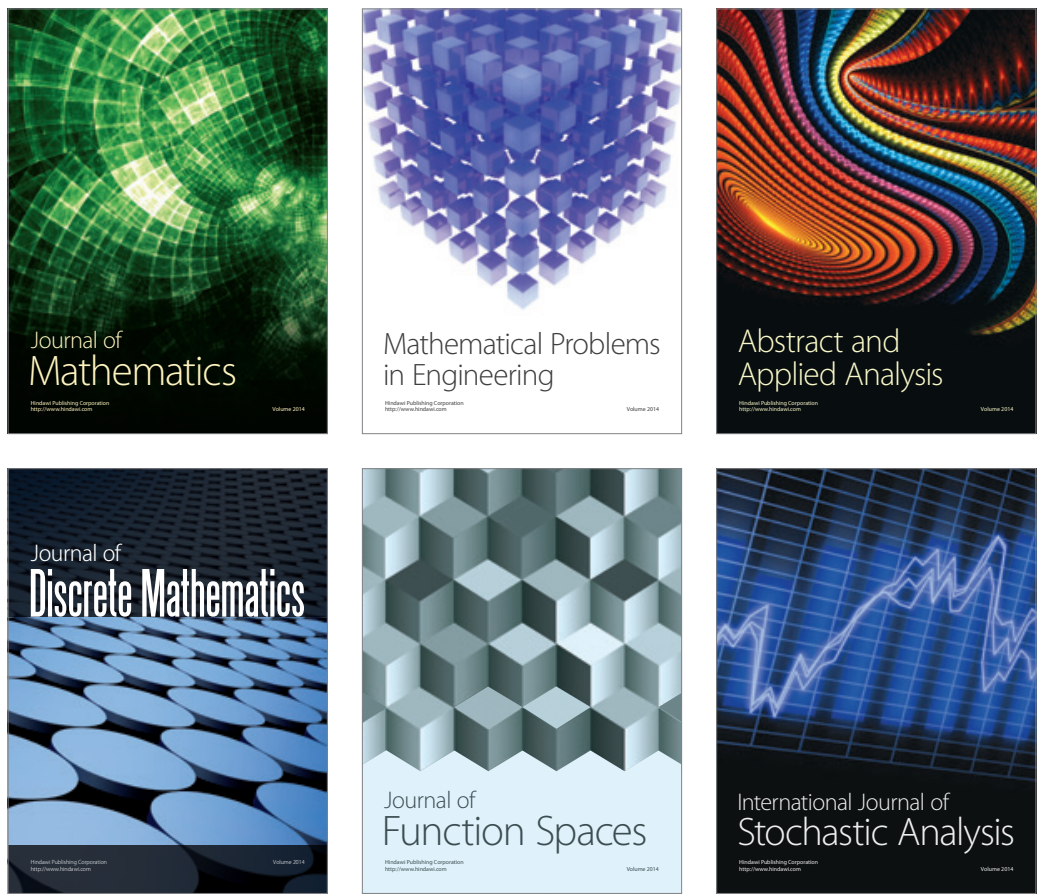

Journal of

Function Spaces

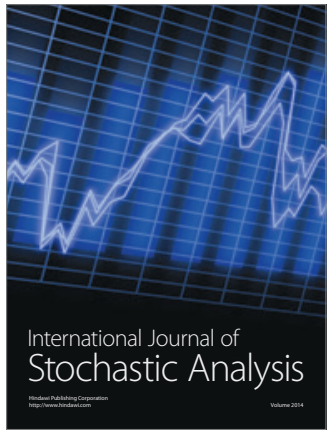

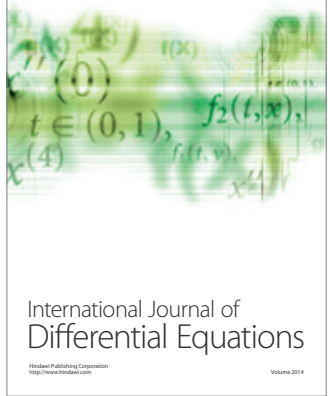
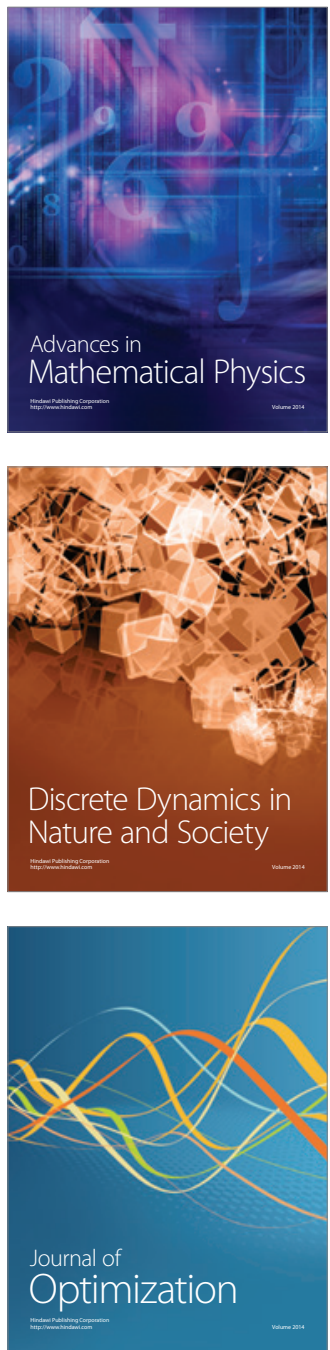